\title{
The National Physical Laboratory Inspection by the General Board
}

\begin{abstract}
THIS annual event was held on June 28, when a large number of visitors, representative of numerous academic, technical and commercial institutions throughout the country were received by Sir William Bragg, chairman of the Board, Lord Rayleigh, chairman of the Executive Committee, and Dr. W. L. Bragg, director of the Laboratory. The Laboratory was thrown open to the visitors and special exhibits demonstrating the work in progress were staged in its various Departments. The time available does not permit the visitor to see all the exhibits, but amongst those of more recent or important the following were noted.
\end{abstract}

\section{Physics Department}

The section of the Physics Department concerned with the thermal properties of materials covers a wide range both of materials and temperatures, some of the extreme examples being typified by apparatus for measuring the thermal conductivity and latent heat of fusion of refrigerants, and another for measuring the thermal properties of steels and other alloys up to temperatures of the order of $1000^{\circ} \mathrm{C}$. The value and convenience of electrical methods of heat production and temperature measurement were well appreciated by a glance through the exhibits in this section.

The Acoustics Section is tackling a number of problems which will eventually contribute to the comfort of a large proportion of the population by the elimination of unnecessary noise. A thorough analysis of the noise emitted by a motor-cycle engine when running under various loads and silencing conditions has begun in a small laboratory which has just been built for these tests. The work on architectural acoustics has largely centred of late on methods of avoiding the transmission of sounds throughout large buildings such as blocks of flats or offices. In one exhibit, a slab of acoustically insulating material such as might be built into the walls of a building was clamped between two plane surfaces one of which was driven at any desired frequency or amplitude. The ratio of the amplitudes of vibration of the driver and driven surfaces was determined for various frequencies and clamping pressures.

The work which has been in progress for several years in the Radiology Section on tooth structure has employed micrographic, radiographic, and X-ray analytical methods of investigation. Tooth enamel has been found to possess a prismatic cell structure, and X-ray analysis has shown that these prisms contain preferentially oriented crystallites of an apatite (probably hydroxy-apatite) the hexagonal axes of which bear a definite angular relation to the axis of the prism in which they lie. The radiographic examination of sections of teeth has also revealed the distribution of their calcium content, and the changes in this distribution consequent on injury or attrition of the enamel at any point have been studied.

A new A.c. bridge circuit has been developed in the Electrical Standards Section, by which capacitance and power factor can be determined in terms of the fundamental standards of mutual inductance and resistance. A completely screened variable substandard mutual inductance incorporating a compensating circuit, which ensures perfect quadrature of secondary E.M.F. and primary current, forms an essential part of the apparatus. The equipment is designed for measurements on capacitances ranging from $500 \mu \mu \mathrm{F}$. to $4 \mu \mathrm{F}$., and the limits of error at 1,000 cycles per second are of the order of \pm 3 parts in $10^{5}$.

The Electrotechnics Section still has a considerable amount of work in hand in connexion with the testing of substandard apparatus for supply authorities throughout Great Britain, and reflecting dynamometer wattmeters have been installed to facilitate the progress of the A.c. instrument testing, which has hitherto been carried out entirely with electrostatic instruments. In the High Voltage Laboratory a new battery of condensers for the generation of transient currents of the order of 100,000 amperes was shown in operation.

A portable telephotometer which has been in use for some years for the measurement of atmospheric transmission over distances up to about a mile was exhibited in the Photometry Section. This instrument is based upon the Maxwellian view principle, and by means of a Lummer-Brodhun prism the intensity of the light received from the distant source is matched by that from a lamp in the instrument. The matching has been found to depend on the size of the image which is formed in the plane of the eye. ring. For reliable measurements, the dimensions of this image should not exceed about $\frac{1}{2} \mathrm{~mm}$.

\section{Radio Department}

Much of the work in the Radio Department is devoted to the higher frequency transmissions which have assumed increasing importance of late. An instrument for the measurement of field-strengths at frequencies of $30 \mathrm{Mc}$. per sec. and upwards consisted of a supersonic-heterodyne receiver, the amplification of which could be adjusted to a reproducible value by operating with a constant 'thermal noise' in the output circuit. By means of a radiator (also exhibited) giving a calculable field, the receiver could then be calibrated at this setting and adjusted to any desired lower sensitivity by the incorporation of calibrated attenuators or voltage dividers in the amplifier. A direction-finding receiver used in conjunction with a cathode ray oscillograph for giving visual indications of bearing was also shown. This incorporated two separate amplifiers of closely identical characteristics-a condition on which the whole success of the apparatus depends.

Another interesting exhibit in this department was a very light-weight short-wave transmitter which has been developed for meteorological work. This apparatus transmits a wave with two modulations, the frequencies of which depend on the atmospheric pressure and temperature respectively, and hence when flown with a sounding balloon this transmitter gives continuous information of changes of these factors on its journey. The complete transmitter 
weighs only $4 \frac{1}{2} \mathrm{lb}$. and has reached heights up to 9 miles and distances of more than 100 miles with good reception throughout.

\section{Metrology Department}

A well-designed and recently completed air-tight chamber for testing mercury barometers was seen in the Metrology Department. This provides accommodation for six barometers, including a standard, and the necessary adjustments for setting each one can be made from outside. The pressure can be varied over a wide range and held constant at any value to within $\pm 0.01 \mathrm{~mm}$. of mercury by a barostat control.

A determination of $g$ by a reversible pendulum has just been completed. The pendulum carries two plane parallel surfaces on which it is swung from a fixed knife-edge on the supporting pillar; an arrangement with which the effective length of the pendulum may be measured with considerably greater accuracy than one in which the pendulum carries knife edges. The effect of the elasticity of the pendulum rod and the finite radius of curvature of the knife edge have been investigated in the course of this work.

In the Gauge-Testing Section a new self-contained vertical type of projector for the examination of plaster casts of ring gauges or of plug gauges up to 3 inches in diameter was shown. This apparatus is transportable and can be used in a normally lighted room.

\section{Engineering Department}

The results of an interesting investigation on 'frettage corrosion', that is, the corrosion which occurs between two metal surfaces which are generally regarded as being in non-rubbing contact, were shown in the Engineering Department. It has been shown that the phenomenon only occurs when an alternating and microscopically small relative move. ment between the two surfaces occurs. Certain combinations of soft and hard metals, especially when lubricated, appear to reduce the corrosion effect, but so far no combination of surfaces has been found to be immune. In the section devoted to the study of lubrication problems, the use of ball-ball and ballplane contacts has enabled reliable measurements to be made under boundary-layer conditions. This has been of particular value in the testing of extreme pressure lubricants. Tests on water-lubricated rubber bearings have shown that the coefficient of friction of a plane cylindrical bearing is considerably lower than that of the more usual fluted type.

A new apparatus for studying the load-relaxation of model pipe flanges at high temperatures was also seen in this Department. This consists of a very rigid frame in which a series assembly of four flanges is compressed by a hydraulic ram. The two centre flanges are thus loaded exactly as in practice, and an extensometer fitted to them measures their total compression. The whole assembly was surrounded by a furnace which could be heated up to $600^{\circ} \mathrm{C}$.

\section{Metallurgy Departient}

The accurate investigation of the iron-carbon, iron-sulphur and iron-nickel systems which is contemplated in the Metallurgy Department has necessitated the production of a regular supply of very pure iron. This is now prepared on a routine basis by chemical methods, using electrolytic iron as the raw material. The chloride is first produced and this is decomposed by steam at a carefully regulated temperature, the oxide so formed being washed free from the chlorides of other metals by water. The dry oxide is reduced in hydrogen and the metal melted, first under slightly oxidizing conditions, then in hydrogen, and finally in vacuo. The final product contains not more than 0.01 per cent of total impurities. The melting of iron without contamination has been made possible by the use of the high-frequency furnace and the production of crucibles of alumina and magnesia.

In this Department there were also two exhibits, one showing the automatic control of the rate of temperature change of a furnace and the other showing the automatic plotting of cooling (or heating) curves, by the combined use of which the tedium usually associated with the accurate determination of transformation points can be avoided entirely. A new gas-fired furnace with an improved design of burner was seen in the foundry. This will attain temperatures up to $1900^{\circ} \mathrm{C}$. and has been used for the firing of the refractory vessels mentioned above and also for testing Seger cones as used in the ceramic industry.

\section{Aerodynamics Department}

In the compressed air tunnel of the Aerodynamies Department a large amount of data upon aerodynamic effects at high Reynolds members has been accumulated. Measurements which are in progress on model wing sections include determinations of drag, of the position of the point of transition from laminar to turbulent flow on the surface, and of pressure distribution over the surface. It will thus be possible to separate skin-friction drag from form drag, and with a knowledge of the transition point, to compare the results with predictions from modern boundary layer theory.

A model of a small airscrew in front of a thick wing was shown in the duplex tunnel. This has been used to investigate the point at which the airscrew becomes so small with respect to the wing that its efficiency begins to fall. The data so obtained will be of value in the design of large aircraft in which these conditions are most likely to occur. The extensive use now being made of kite balloons has stimulated further interest in their stability and a model of a balloon was seen undergoing tests on the whirling arm.

\section{Froude Laboratory}

In the Yarrow Tank of the Froude Laboratory a tug propelled by twin paddle wheels, towing two barges, was under test. Power and speed measurements were automatically recorded, and the method of reduction to ship dimensions was explained. A model of a high-speed hull was demonstrated in the new tank. This type of work is of growing importance owing to the increasing use of small speed-boats for naval and commercial purposes.

In conclusion, a tribute might well be made here to the garden staff, which has created and maintained the pleasant surroundings in which the Laboratory is set and which help to make a visit on such an occasion as this a delight as well as an interest. 